IASSNS-HEP-97/15

March, 1997

\title{
The Matrix Model for M Theory as an Exemplar of Trace (or Generalized Quantum) Dynamics
}

\author{
Stephen L. Adler \\ Institute for Advanced Study \\ Princeton, NJ 08540
}

Send correspondence to:

Stephen L. Adler

Institute for Advanced Study

Olden Lane, Princeton, NJ 08540

Phone 609-734-8051; FAX 609-924-8399; email adler@ias. edu 


\begin{abstract}
We show that the recently proposed matrix model for M theory obeys the cyclic trace assumptions underlying generalized quantum or trace dynamics. This permits a verification of supersymmetry as an operator calculation, and a calculation of the supercharge density algebra by using the generalized Poisson bracket, in a basis-independent manner that makes no reference to individual matrix elements. Implications for quantization of the model are discussed. Our results are a special case of a general result presented elsewhere, that all rigid supersymmetry theories can be extended to give supersymmetric trace dynamics theories, in which the supersymmetry algebra is represented by the generalized Poisson bracket of trace supercharges, constructed from fields that form a noncommutative trace class graded operator algebra.
\end{abstract}


Recently Banks, Fischler, Shenker, and Susskind [1] have suggested a supersymmetric matrix model (based on earlier supersymmetric quantum mechanics models [2]) for use as a tool to study uncompactified eleven dimensional $M$ theory, and more recently Banks, Seiberg, and Shenker [3] have computed the supersymmetry charge density algebra in this model. Although they performed this computation using the Poisson brackets for the individual matrix elements, the fact that the final answers are expressible directly in matrix terms suggests that it should be possible to perform the calculation using matrix or operator methods throughout. We shall show in this paper that the operator dynamics that we have proposed [4] and studied with various collaborators $[5,6]$ is admirably suited for this purpose. In the original papers we termed this operator dynamics "generalized quantum dynamics", but A. Millard in his thesis [7] uses the briefer and more descriptive name "trace dynamics", which we shall use henceforth. What we shall show here is that trace dynamics permits an operator treatment of the matrix model for M theory, and generalizes this model to the case in which the matrix elements are themselves noncommutative matrices (as would be of interest for renormalization group block spin schemes). More generally, we shall show elsewhere [8] that all rigid supersymmetry theories can be extended to supersymmetric trace dynamics theories, giving supersymmetry representations over trace class noncommutative graded operator algebras. Because of the current interest in the model of [1], and the fact that this model does not require the gauge fixing technicalities that enter into the case of supersymmetric Yang-Mills theory, it is well suited to a brief account illustrative of our general approach.

Let $X_{1}$ and $X_{2}$ be two $N \times N$ matrices with complex (or more generally, complex Grassmann even) matrix elements, and Tr the ordinary matrix trace, which obeys the cyclic 
property

$$
\operatorname{Tr} X_{1} X_{2}=\sum_{m, n}\left(X_{1}\right)_{m n}\left(X_{2}\right)_{n m}=\sum_{m, n}\left(X_{2}\right)_{n m}\left(X_{1}\right)_{m n}=\operatorname{Tr} X_{2} X_{1}
$$

Correspondingly, let $\theta_{1}$ and $\theta_{2}$ be two $N \times N$ matrices with complex Grassmann odd matrix elements, which anticommute rather than commute, so that the cyclic property for these takes the form

$$
\operatorname{Tr} \theta_{1} \theta_{2}=\sum_{m, n}\left(\theta_{1}\right)_{m n}\left(\theta_{2}\right)_{n m}=-\sum_{m, n}\left(\theta_{2}\right)_{n m}\left(\theta_{1}\right)_{m n}=-\operatorname{Tr} \theta_{2} \theta_{1}
$$

The cyclic properties of Eqs. (1a, 1b) are just those assumed for the trace operation $\operatorname{Tr}$ of trace dynamics (although in Refs. [4-6] the fermionic operators are realized as matrices with complex matrix elements, all of which anticommute with a grading operator $\left.(-1)^{F}\right)$; we shall continue here to use the notation Tr, with the understanding that fermionic matrices are Grassmann odd matrices obeying Eq. (1b), while bosonic matrices are Grassmann even matrices obeying Eq. (1a). From Eqs. (1a) and (1b), one immediately derives the trilinear cyclic identities

$$
\begin{gathered}
\operatorname{Tr} X_{1}\left[X_{2}, X_{3}\right]=\operatorname{Tr} X_{2}\left[X_{3}, X_{1}\right]=\operatorname{Tr} X_{3}\left[X_{1}, X_{2}\right] \\
\operatorname{Tr} X_{1}\left\{X_{2}, X_{3}\right\}=\operatorname{Tr} X_{2}\left\{X_{3}, X_{1}\right\}=\operatorname{Tr} X_{3}\left\{X_{1}, X_{2}\right\} \\
\operatorname{Tr} X\left\{\theta_{1}, \theta_{2}\right\}=\operatorname{Tr} \theta_{1}\left[\theta_{2}, X\right]=\operatorname{Tr} \theta_{2}\left[\theta_{1}, X\right] \\
\operatorname{Tr} \theta_{1}\left\{X, \theta_{2}\right\}=\operatorname{Tr}\left\{\theta_{1}, X\right\} \theta_{2}=\operatorname{Tr}\left[\theta_{1}, \theta_{2}\right] X
\end{gathered}
$$

which are repeatedly used below.

The basic observation of trace dynamics is that given the trace of a polynomial $P$ constructed from noncommuting operator variables, one can define a derivative of the number $\operatorname{Tr} P$ with respect to an operator variable $\mathcal{O}$ by varying and then cyclically permuting so that 
in each term the factor $\delta \mathcal{O}$ stands on the right, giving the fundamental definition

$$
\delta \operatorname{Tr} P=\operatorname{Tr} \frac{\delta \operatorname{Tr} P}{\delta \mathcal{O}} \delta \mathcal{O}
$$

or in the condensed notation with $\mathbf{P} \equiv \operatorname{Tr} P$,

$$
\delta \mathbf{P}=\operatorname{Tr} \frac{\delta \mathbf{P}}{\delta \mathcal{O}} \delta \mathcal{O}
$$

In Refs. $[4,5]$ it is shown that using this definition, one can construct a complete Lagrangian and Hamiltonian dynamics for systems with noncommuting graded operator variables and a trace Lagrangian, in which a generalized Poisson bracket plays the role played in classical mechanics by the classical Poisson bracket, or by the commutator in quantum mechanics. In Ref. [6] it is further shown that this dynamical system has the remarkable property that its statistical mechanics gives complex quantum field theory, with ensemble averages of the operator variables effectively obeying standard canonical commutation relations.

Let us now turn to the matrix model for $M$ theory. It is described by the trace Lagrangian $\mathbf{L}$ given by

$$
\mathbf{L}=\operatorname{Tr}\left(\frac{1}{2} D_{t} X_{i} D_{t} X^{i}+i \theta^{T} D_{t} \theta+\frac{1}{4}\left[X_{i}, X_{j}\right]\left[X^{i}, X^{j}\right]+\theta^{T} \gamma_{i}\left[\theta, X^{i}\right]\right)
$$

with $D_{t} \mathcal{O}=\partial_{t} \mathcal{O}-i\left[A_{0}, \mathcal{O}\right]$. In Eq. (3a), a summation convention is understood on the indices $i, j$ which range from 1 to $9 ; A_{0}$ and the $X_{i}$ are self-adjoint $N \times N$ bosonic matrices with complex number matrix elements, while $\theta$ is a 16 -component fermionic spinor each element of which is a self-adjoint $N \times N$ complex Grassmann matrix, with the transpose $T$ acting only on the spinor structure but not on the $N \times N$ matrices, so that $\theta^{T}$ is simply the 16 component row spinor corresponding to the 16 component column spinor $\theta$. The potential $A_{0}$ has no kinetic term and so is a pure gauge degree of freedom. Finally, the $\gamma_{i}$ are a set of 
nine $16 \times 16$ matrices, which are related to the standard $32 \times 32$ matrices $\Gamma_{\mu}$ as well as to the Dirac matrices of $\operatorname{spin}(8)$ as described in Danielsson, Ferretti, and Sundborg [9]. This finishes the specification of the model.

Before turning to a study of the model's dynamics and supersymmetries, let us summarize some of the properties of the real, symmetric matrices $\gamma_{i}$ that are needed. These matrices satisfy the anticommutator algebra

$$
\left\{\gamma_{i}, \gamma_{j}\right\}=2 \delta_{i j}
$$

as well as the cyclic identity (which follows by projection from Eq. (4.A.6) of Green, Schwarz, and Witten [10]),

$$
\sum_{\text {cycle }}\left(\delta^{m n} \delta^{p q}-\gamma_{i}^{m n} \gamma_{i}^{p q}\right)=0 \quad,
$$

with $i$ again summed over and with the indices $m, n, p, q$ spinorial indices ranging from 1 to 16. Defining

$$
\gamma_{i j}=\frac{1}{2}\left[\gamma_{i}, \gamma_{j}\right]
$$

so that

$$
\gamma_{i} \gamma_{j}=\delta_{i j}+\gamma_{i j}
$$

one readily derives from Eq. (4b) an identity given in Ref. [3],

$$
\gamma_{i j}^{m n} \gamma_{i}^{p q}+\gamma_{i j}^{p q} \gamma_{i}^{m n}+(m \leftrightarrow p)=2\left(\gamma_{j}^{n q} \delta^{m p}-\gamma_{j}^{m p} \delta^{n q}\right) \quad .
$$

By standard gamma matrix manipulations using Eq. (4a), one also derives the the fact that the matrix

$$
A_{i j k}=\gamma_{i} \gamma_{j} \gamma_{k}-\delta_{i j} \gamma_{k}+\delta_{i k} \gamma_{j}-\delta_{j k} \gamma_{i}
$$


is totally antisymmetric in the indices $i, j, k$ (it is just the antisymmetrized product $\gamma_{[i} \gamma_{j} \gamma_{k]}$ with normalization factor $\frac{1}{6}$ ), as well as the identity

$$
\frac{1}{2}\left\{\gamma_{\ell m}, \gamma_{i j}\right\}=\gamma_{[\ell} \gamma_{m} \gamma_{i} \gamma_{j]}+\delta_{\ell j} \delta_{i m}-\delta_{m j} \delta_{i \ell}
$$

with the first term on the right the antisymmetrized product including normalization factor $\frac{1}{24}$.

Now let us turn to dynamics. From the trace Lagrangian of Eq. (3), using the definition of Eq. (2) we find the operator derivatives

$$
\begin{aligned}
\frac{\delta \mathbf{L}}{\delta A_{0}} & =-i\left[X^{i}, D_{t} X_{i}\right]-2 \theta^{T} \theta \\
\frac{\delta \mathbf{L}}{\delta X_{i}} & =-i\left[D_{t} X^{i}, A_{0}\right]+\left[\left[X^{j}, X^{i}\right], X_{j}\right]+2 \theta^{T} \gamma^{i} \theta \\
\frac{\delta \mathbf{L}}{\delta\left(\partial_{t} X_{i}\right)} & =D_{t} X^{i} \\
\frac{\delta \mathbf{L}}{\delta \theta} & =-i D_{t} \theta^{T}+\left[\theta^{T}, A_{0}\right]-2\left[\theta^{T} \gamma_{i}, X^{i}\right] \\
\frac{\delta \mathbf{L}}{\delta\left(\partial_{t} \theta\right)} & =i \theta^{T}
\end{aligned}
$$

Substituting these into the operator Euler-Lagrange equations [4]

$$
\frac{\partial}{\partial t} \frac{\delta \mathbf{L}}{\delta\left(\partial_{t} \mathcal{O}\right)}=\frac{\delta \mathbf{L}}{\delta \mathcal{O}}
$$

and regrouping terms, we get the equations of motion of the matrix model

$$
\begin{aligned}
D_{t}^{2} X^{i} & =\left[\left[X^{j}, X^{i}\right], X_{j}\right]+2 \theta^{T} \gamma^{i} \theta \\
D_{t} \theta^{T} & =i\left[\theta^{T} \gamma_{i}, X^{i}\right] \Rightarrow D_{t} \theta=i\left[\gamma_{i} \theta, X^{i}\right],
\end{aligned}
$$

together with the constraint

$$
\tilde{C} \equiv\left[X^{i}, D_{t} X_{i}\right]-2 i \theta^{T} \theta=0
$$

To transform the dynamics to trace Hamiltonian form, we define the canonical momenta $p_{X_{i}}$ 
and $p_{\theta}$ by

$$
\begin{aligned}
p_{X_{i}} & =\frac{\delta \mathbf{L}}{\delta\left(\partial_{t} X_{i}\right)}=D_{t} X^{i} \\
p_{\theta} & =\frac{\delta \mathbf{L}}{\delta\left(\partial_{t} \theta\right)}=i \theta^{T}
\end{aligned}
$$

so that the trace Hamiltonian is given by

$\mathbf{H}=\operatorname{Tr}\left(p_{X_{i}} \partial_{t} X_{i}+p_{\theta} \partial_{t} \theta\right)-\mathbf{L}=\operatorname{Tr}\left(\frac{1}{2} p_{X_{i}} p_{X^{i}}-\frac{1}{4}\left[X_{i}, X_{j}\right]\left[X^{i}, X^{j}\right]+i p_{\theta} \gamma_{i}\left[\theta, X^{i}\right]+i A_{0} \tilde{C}\right)$

Note that because $p_{\theta}=i \theta^{T}$, it is necessary to write the trace Hamiltonian in a form that is manifestly symmetric under the replacements $p_{\theta} \rightarrow i \theta^{T}, \quad \theta \rightarrow-i p_{\theta}^{T}$; it is easy to check that by virtue of the cyclic identities of Eq.(1c) and the symmetry of $\gamma_{i}$ that Eq. (8b) has this symmetry. The Hamilton equations following from the trace Hamiltonian of Eq. (8b) are [4]

$$
\begin{aligned}
\partial_{t} X_{i} & =\frac{\delta \mathbf{H}}{\delta p_{X_{i}}} \\
\partial_{t} p_{X_{i}} & =-\frac{\delta \mathbf{H}}{\delta X_{i}} \\
\partial_{t} \theta & =-\frac{\delta \mathbf{H}}{\delta p_{\theta}} \\
\partial_{t} p_{\theta} & =-\frac{\delta \mathbf{H}}{\delta \theta}
\end{aligned}
$$

and it is easy to check that they are the same as the operator Euler-Lagrange equations derived above.

So far we have reproduced standard results of the matrix model, but have broken no new ground. Now let us consider the variation of the trace Lagrangian, calculated from

$$
\delta \mathbf{L}=\operatorname{Tr}\left(\frac{\delta \mathbf{L}}{\delta A_{0}} \delta A_{0}+\frac{\delta \mathbf{L}}{\delta\left(\partial_{t} X_{i}\right)} \delta\left(\partial_{t} X_{i}\right)+\frac{\delta \mathbf{L}}{\delta X_{i}} \delta X_{i}+\frac{\delta \mathbf{L}}{\delta\left(\partial_{t} \theta\right)} \delta\left(\partial_{t} \theta\right)+\frac{\delta \mathbf{L}}{\delta \theta} \delta \theta\right)
$$


for the supersymmetry transformation defined by

$$
\begin{aligned}
\delta X^{i} & =-2 \epsilon^{T} \gamma^{i} \theta=2 \theta^{T} \gamma^{i} \epsilon \\
\delta \theta & =-\left(i D_{t} X^{i} \gamma_{i}+\frac{1}{2}\left[X^{i}, X^{j}\right] \gamma_{i j}\right) \epsilon+\epsilon^{\prime} \\
\delta A_{0} & =-2 \epsilon^{T} \theta=2 \theta^{T} \epsilon .
\end{aligned}
$$

Here $\epsilon$ and $\epsilon^{\prime}$ are 16 component Grassmann $c$-number spinors, that is, they are column vectors each of whose 16 components is an independent $1 \times 1$ Grassmann matrix. Using only the cyclic trace identities and gamma matrix properties given above, it is a matter of straightforward but lengthy calculation to verify that the trace Lagrangian is invariant under the transformation of Eq. (10b) when $\epsilon$ and $\epsilon^{\prime}$ are time independent. Note that in this calculation the variables $X_{i}, \theta$, and $A_{0}$ are treated simply as noncommuting operators that are unspecified apart from their bosonic or fermionic character; in particular, we do not have to assume that their matrix elements when they are written as $N \times N$ matrices are $c$-numbers or Grassmann $c$-numbers. In other words, we have shown that the matrix model of Eq. (3) is still supersymmetric when viewed as a model over general noncommutative trace class graded operator variables.

When $\epsilon$ has a time dependence $\delta \mathbf{L}$ is no longer zero, but instead is given by

$$
\begin{aligned}
\delta \mathbf{L} & =\partial_{t} \operatorname{Tr}\left[-i \theta^{T} \epsilon^{\prime}+\left(\theta^{T} \gamma_{i} D_{t} X^{i}+\frac{1}{2} i \theta^{T} \gamma_{i j}\left[X^{i}, X^{j}\right]\right) \epsilon\right] \\
& +\operatorname{Tr}\left[2 i \theta^{T} \partial_{t} \epsilon^{\prime}+\left(2 \theta^{T} \gamma_{i} D_{t} X^{i}-i \theta^{T} \gamma_{i j}\left[X^{i}, X^{j}\right]\right) \partial_{t} \epsilon\right]
\end{aligned}
$$

This identifies the trace supercharges $\mathbf{Q}_{\alpha}^{\prime}$ and $\mathbf{Q}_{\alpha}$ as

$$
\begin{aligned}
& \mathbf{Q}_{\alpha}^{\prime}=\operatorname{Tr} 2 i \theta^{T} \alpha \\
& \mathbf{Q}_{\alpha}=\operatorname{Tr}\left(2 \theta^{T} \gamma_{i} D_{t} X_{i}-i \theta^{T} \gamma_{i j}\left[X^{i}, X^{j}\right]\right) \alpha,
\end{aligned}
$$

and their conservation is easily checked using the equations of motion and constraint of Eqs. (8a, b) and the identities of Eqs. $(4,5)$. To check the supersymmetry algebra, we must 
first write the supercharges of Eq. (11b) in Hamiltonian form, symmetrized with respect to $p_{\theta}$ and $i \theta^{T}$, giving

$$
\begin{aligned}
& \mathbf{Q}_{\alpha}^{\prime}=\operatorname{Tr}\left(p_{\theta}+i \theta^{T}\right) \alpha \\
& \mathbf{Q}_{\alpha}=-\operatorname{Tr}\left(p_{\theta}+i \theta^{T}\right)\left(i \gamma_{i} p_{X_{i}}+\frac{1}{2} \gamma_{i j}\left[X^{i}, X^{j}\right]\right) \alpha .
\end{aligned}
$$

In addition to the supercharge $\mathbf{Q}_{\alpha}$, Ref. [3] also introduces a supercharge density; in our language this can be written in terms of a Grassmann spinor operator $\beta$ as

$$
\mathbf{Q}_{\beta}=-\frac{1}{2} \operatorname{Tr}\left\{\left(p_{\theta}+i \theta^{T}\right), i \gamma_{i} p_{X_{i}}+\frac{1}{2} \gamma_{i j}\left[X^{i}, X^{j}\right]\right\} \beta
$$

Let us now introduce the generalized Poisson bracket $[4,5]$ corresponding to the Hamiltonian structure of our model, defined for any $\mathbf{A}=\operatorname{Tr} A$ and $\mathbf{B}=\operatorname{Tr} B$ by

$$
\{\mathbf{A}, \mathbf{B}\}=\operatorname{Tr}\left(\frac{\delta \mathbf{A}}{\delta X_{i}} \frac{\delta \mathbf{B}}{\delta p_{X_{i}}}-\frac{\delta \mathbf{B}}{\delta X_{i}} \frac{\delta \mathbf{A}}{\delta p_{X_{i}}}-\frac{\delta \mathbf{A}}{\delta \theta} \frac{\delta \mathbf{B}}{\delta p_{\theta}}+\frac{\delta \mathbf{B}}{\delta \theta} \frac{\delta \mathbf{A}}{\delta p_{\theta}}\right)
$$

We can now use the generalized Poisson bracket to give a basis-independent evaluation of the supersymmetry charge algebra. For the brackets involving $\mathbf{Q}^{\prime}$, we easily find

$$
\begin{aligned}
& \left\{\mathbf{Q}_{\alpha}^{\prime}, \mathbf{Q}_{\beta}^{\prime}\right\}=-2 i \operatorname{Tr} \alpha \beta \\
& \left\{\mathbf{Q}_{\alpha}^{\prime}, \mathbf{Q}_{\beta}\right\}=2 i \operatorname{Tr}\left(i \gamma_{i}^{a b} p_{X_{i}}+\frac{1}{2} \gamma_{i j}^{a b}\left[X^{i}, X^{j}\right]\right) \alpha_{a} \beta_{b}
\end{aligned}
$$

The only case involving significant work is the bracket of $\mathbf{Q}_{\alpha}$ with $\mathbf{Q}_{\beta}$, which on substituting the operator derivatives of the Q's into Eq. (13), but before further algebraic rearrangement, takes the form

$$
\left\{\mathbf{Q}_{\alpha}, \mathbf{Q}_{\beta}\right\}=\operatorname{Tr} U
$$


with $U$ given by

$$
\begin{aligned}
U & =2\left[X^{j}, \alpha_{b} 2 i \theta_{a} \frac{1}{2} \gamma_{i j}^{a b}\right]\left[\beta_{d}, i \theta_{c}\right] i \gamma_{i}^{c d} \\
& +\left[X^{m},\left[2 i \theta_{c}, \beta_{d}\right]\right] \frac{1}{2} \gamma_{i m}^{c d} \alpha_{b} 2 i \theta_{a} i \gamma_{i}^{a b} \\
& -i\left(i \gamma_{i}^{a b} p_{X_{i}}+\frac{1}{2} \gamma_{i j}^{a b}\left[X^{i}, X^{j}\right]\right) \alpha_{b} \frac{1}{2}\left\{\beta_{d}, i \gamma_{\ell}^{a d} p_{X_{\ell}}+\frac{1}{2} \gamma_{\ell m}^{a d}\left[X^{\ell}, X^{m}\right]\right\} \\
& +\frac{1}{2} i\left\{\beta_{d}, i \gamma_{\ell}^{a d} p_{X_{\ell}}+\frac{1}{2} \gamma_{\ell m}^{a d}\left[X^{\ell}, X^{m}\right]\right\}\left(i \gamma_{i}^{a b} p_{X_{i}}+\frac{1}{2} \gamma_{i j}^{a b}\left[X^{i}, X^{j}\right]\right) \alpha_{b}
\end{aligned}
$$

Algebraic rearrangement of this using the cyclic and gamma matrix identities gives $\operatorname{Tr} U=$ $\operatorname{Tr} T$

$$
T=T_{1} 4 i \delta^{b d} \alpha_{b} \beta_{d}+T_{2}^{j} 2 \gamma_{j}^{b d} \alpha_{b} \beta_{d}+X^{[\ell} X^{m} X^{i} X^{j]} 2 i\left(\gamma_{[\ell} \gamma_{m} \gamma_{i} \gamma_{j]}\right)^{b d} \alpha_{b} \beta_{d}
$$

with

$$
\begin{aligned}
T_{1} & =\frac{1}{2} p_{X^{\ell}} p_{X_{\ell}}-\frac{1}{4}\left[X_{\ell}, X_{m}\right]\left[X^{\ell}, X^{m}\right]+\frac{1}{2}\left[\theta_{a},\left[X^{j}, \theta_{c}\right]\right] \gamma_{j}^{a c} \\
\operatorname{Tr} T_{1} & =\mathbf{H}
\end{aligned}
$$

and

$$
\begin{aligned}
T_{2}^{j} & =\left\{p_{X^{i}},\left[X^{i}, X^{j}\right]\right\}-i\left[\theta_{a},\left[X^{j}, \theta^{a}\right]\right] \\
& =-\left\{X^{j}, \tilde{C}\right\}+\left[X^{i},\left\{p_{X^{i}}, X^{j}\right\}\right]-i\left\{\theta_{a},\left\{X^{j}, \theta^{a}\right\}\right\},
\end{aligned}
$$

where in getting the final line of Eq. (16c) we have used the Jacobi identities for mixed commutators and anticommutators. Equations (14) and (16a-c) agree with the charge density algebra computed in Ref. [3]. In the case when $\beta$ is a $c$-number, we see that the generalized Poisson bracket of the trace supercharges gives the trace Hamiltonian, showing that we have constructed a supersymmetry representation over noncommutative trace class operator dynamical variables. As noted earlier, we will show elsewhere [8] that this construction works for general rigid supersymmetric theories, and in particular for the Wess-Zumino and supersymmetric Yang-Mills theories in four dimensions.

We close with a remark on the quantization of the matrix model just described. There are two possible points of view one could take. The first possibility would be to 
quantize by replacing the Poisson brackets for matrix elements used in the computations of Ref. [3] by a commutator/anticommutator algebra; in this case one would be treating the matrix elements themselves as quantum operators. An alternative possibility is discussed in Ref. [6], which is to regard the trace dynamics as fundamental without quantization, and to consider its statistical mechanics assuming ergodicity (an assumption that may presuppose taking the large $N$ limit; we also remark that the proof of the generalized Liouville theorem in Ref. [6] extends, with minor modifications, to the case

employed here in which the fermions are realized with Grassmann matrices.) It is then shown that the statistical averages of the dynamical variables obey the rules of complex quantum field theory, with the effective Planck constant given by the expectation of the conserved operator $\tilde{C}$ discovered by Millard [7], which for a generic trace dynamics model has the form

$$
\tilde{C}=\sum_{\text {bosons }}\left[q_{i}, p_{i}\right]-\sum_{\text {fermions }}\left\{q_{i}, p_{i}\right\}
$$

Since for the trace Lagrangian of Eq. (3) $\tilde{C}$ is just the expression of Eq. (7b), which vanishes as a constraint, the analysis of Ref. [6] implies that the statistical averages will obey classical mechanics, with the $X_{i}$ all commuting with one another and with the corresponding $p_{X_{j}}$. As discussed in Ref. [4], it is easy to modify the model so that the constraint of Eq. (7b) reads instead $\tilde{C}=i \hbar$; this is done by adding to $\mathbf{L}$ the term

$$
\Delta \mathbf{L}=-\operatorname{Tr} A_{0}
$$

which gives a trace action $\Delta \mathbf{S}=\int d t \mathbf{L}$ that is invariant under gauge transformations that vanish (or are periodic) at $t= \pm \infty$. As we shall show in Ref. [8], it is easy to construct supersymmetric trace dynamics theories in which the operator $\tilde{C}$, although conserved, is 
not constrained to vanish; the trace dynamics extension of the Wess-Zumino model is an example.

\section{Acknowledgments}

This work was supported in part by the Department of Energy under Grant \#DEFG02-90ER40542. I wish to thank Ed Witten, Andrew Millard, and members of the Princeton graduate student supersymmetry discussion group, for useful conversations. 


\section{References}

[1] T. Banks, W. Fischler, S. H. Shenker, and L. Susskind, "M Theory as A Matrix Model: A Conjecture", hep-th/9610043.

[2] M. Claudson and M. B. Halpern, Nucl. Phys. B250 (1985) 689; V. Rittenberg and S. Yankielowicz, Ann. Phys. 162 (1985) 273; R. Flume, Ann. Phys. 164 (1985) 189. For a recent survey, see B. de Wit, "Supersymmetric quantum mechanics, supermembranes and Dirichlet particles", hep-th/9701169.

[3] T. Banks, N. Seiberg, and S. Shenker, "Branes from Matrices", hep-th/9612157.

[4] S. L. Adler, Nucl. Phys. B 415 (1994) 195; S. L. Adler, "Quaternionic Quantum Mechanics and Quantum Fields", Sects. 13.5-13.7 and App. A (Oxford Univ. Press, New York, 1995).

[5] S. L. Adler, G. V. Bhanot, and J. D. Weckel, J. Math. Phys. 35 (1994), 531; S. L. Adler and Y.-S. Wu, Phys. Rev. D 49 (1994) 6705.

[6] S. L. Adler and A. C. Millard, Nucl. Phys. B 473 (1996) 199.

[7] A. C. Millard, Princeton University PhD thesis (in preparation).

[8] S. L. Adler, "Poincaré Supersymmetry Representations Over Trace Class Noncommutative Graded Operator Algebras", IASSNS-HEP-97/16 (in preparation).

[9] U. H. Danielsson, G. Ferretti, and B. Sundborg, "D-Particle Dynamics and Bound States", hep-th/9603081. 
[10] M. B. Green, J. H. Schwarz, and E. Witten, "Superstring Theory", Vol. 1, p.246 (Cambridge Univ. Press, Cambridge, 1987). 